\title{
Tendências de Incidência e Mortalidade por Câncer de Ovário nos Países da América Latina
}

doi: https://doi.org/10.32635/2176-9745.RBC.2020v66n4.813

\author{
Trends in Incidence and Mortality by Ovarian Cancer in Latin American Countries \\ Tendencias de Incidencia y Mortalidad por Cáncer de Ovario en Países de América Latina
}

\author{
Maria Aparecida Paulo dos Santos'; Fábia Cheyenne Gomes de Morais Fernandes²; Emelynne Gabrielly de Oliveira Santos ${ }^{3}$; Dyego \\ Leandro Bezerra de Souza ${ }^{4}$; Isabelle Ribeiro Barbosa ${ }^{5}$
}

RESUMO

Introduçáo: $\mathrm{O}$ câncer de ovário é o sétimo mais comum e a nona causa de morte por câncer em mulheres, no mundo. Na América Latina, o câncer é a segunda causa de morte, e as projeçôes indicam um provável crescimento no número de mortes entre os anos 2012 e 2035. Objetivo: Analisar as tendências de incidência e mortalidade para o câncer de ovário em países da América Latina. Método: Estudo ecológico de série temporal, com dados de incidência extraídos da Agência Internacional para Pesquisa do Câncer de 1990 a 2012 , e dados de mortalidade obtidos de 17 países da Organização Mundial da Saúde de 1995 a 2013. A tendência de incidência foi analisada pela regressão Joinpoint. A variação percentual média anual (AAPC) e o intervalo de confiança de $95 \%$ foram calculados para incidência e mortalidade. Resultados: As maiores taxas de incidência para o câncer de ovário foram observadas em Cali (Colômbia) e Goiânia (Brasil) na faixa etária acima de 60 anos. Houve tendência de estabilidade na maioria dos países, exceto em Goiânia (Brasil), na faixa etária de 60-74 anos, que apresentou tendência de aumento (AAPC: 3,4\%; IC 95\%: 0,3; 6,6). A maior taxa de mortalidade por câncer de ovário ocorreu no Uruguai para a faixa etária de 60-74 anos e a menor, em Belize e Guatemala, para a faixa etária de 0-39 anos. Conclusáo: A incidência do câncer de ovário na América Latina apresentou estabilidade na maioria dos países. Em alguns, como o Brasil, observou-se aumento nas tendências de mortalidade.

Palavras-chave: Neoplasias Ovarianas/epidemiologia; Neoplasias Ovarianas/mortalidade; Incidência; América Latina/epidemiologia.

\section{ABSTRACT}

Introduction: Ovarian cancer is the seventh most common and the ninth cause of death by cancer in women worldwide. In Latin America, cancer is the second cause of death and projections indicate a probable growth of the number of deaths between 2012 and 2035. Objective: To analyze the trends of incidence and mortality by ovarian cancer in Latin American countries. Method: Ecological time series study, with incidence data extracted from the International Agency for Research on Cancer, from 1990 to 2012 and mortality data from 17 countries of the World Health Organization, from 1995 to 2013. An incidence trend was analyzed by the Joinpoint regression. The average annual percentage change (AAPC) and the 95\% confidence interval were calculated for incidence and mortality. Results: The highest incidence rates of ovarian cancer were observed in Cali (Colombia) and Goiânia (Brazil) in the age group older than 60 years. There was a tendency towards stability in most countries, except in Goiânia (Brazil), in the age group from 60-74 years old, with an increasing trend (AAPC: 3.4\%; 95\% CI: $0.3 ; 6.6)$. The highest rate of mortality by ovarian cancer occurred in Uruguay for the age group of 60-74 years and the lowest, in Belize and Guatemala, for the age group of 0-39 years. Conclusion: The incidence of ovarian cancer in Latin America has remained stable in most countries. In some, as Brazil, increase of mortality rates was observed.

Key words: Ovarian Neoplasms/epidemiology; Ovarian Neoplasms/ mortality; Incidence; Latin America/epidemiology.

\section{RESUMEN}

Introducción: el cáncer de ovario es el más común y la novena causa de muerte por cáncer en mujeres de todo el mundo. En América Latina, el cáncer es la segunda causa principal de muerte y las proyecciones se consideran un número probable de muertes entre los ańos 2012 y 2035. Objetivo: Analizar las tendencias de mortalidad y mortalidad por cáncer de ovario en los países latinoamericanos. Método: Estudio de series de tiempo ecológicas, con datos extraídos por la Agencia Internacional de Investigación sobre el Cáncer, de 1990 a 2012 y datos con mortalidad de 17 países de la Organización Mundial de la Salud, de 1995 a 2013. Se analizó una tendencia de incidencia mediante la regresión de Joinpoint. El cambio porcentual anual medio (AAPC) y el intervalo de confianza del $95 \%$ se calcularon para la incidencia y la mortalidad. Resultados: Las tasas de incidencia más altas de cáncer de ovario se observaron en Cali (Colombia) y Goiânia (Brasil) en el grupo de edad de más de 60 años. Hubo una tendencia hacia la estabilidad en la mayoría de los países, excepto en Goiânia (Brasil), en el grupo de edad de 60-74 años, con una tendencia creciente (AAPC: 3,4\%; IC 95\%: 0,3; 6,6). Las tasas de mortalidad más altas debidas al cáncer de ovario no ocurrieron en Uruguay para el grupo de edad de 60-74 años y las más bajas en Belice y Guatemala, para el grupo de edad de 0-39 años. Conclusión: La incidencia del cáncer de ovario en América Latina se ha mantenido estable en la mayoría de los países. En algunos, como Brasil, las tendencias de mortalidad pueden aumentar.

Palabras clave: Neoplasias Ováricas/epidemiología; Neoplasias Ováricas/ mortalidade; Incidencia; América Latina/epidemiología.

${ }^{1}$ Faculdade de Ciências da Saúde do Trairi (Facisa). Universidade Federal do Rio Grande do Norte (UFRN). Natal (RN), Brasil. Orcid iD: https://orcid.org/0000-00026997-8168

${ }^{2}$ Facisa. UFRN. Natal (RN), Brasil. Orcid iD: http://orcid.org/0000-0002-0834-855X

${ }^{3}$ Facisa. UFRN. Natal (RN), Brasil. Orcid iD: http://orcid.org/0000-0002-3890-4879

${ }^{4}$ Departamento de Saúde Coletiva da UFRN. Natal (RN), Brasil. Orcid iD: https://orcid.org/0000-0001-8426-3120

${ }^{5}$ Facisa. UFRN. Natal (RN), Brasil. Orcid iD: http://orcid.org/0000-0002-1385-2849

Endereço para correspondência: Emelynne Gabrielly de Oliveira Santos. Rua Joaquim Inácio, 1375 - Tirol. Natal (RN), Brasil. CEP 59022-180.

E-mail: emeoliveirasantos@hotmail.com 


\section{INTRODUÇÃO}

O câncer de ovário é o sétimo câncer mais comum e a nona causa de morte por câncer em mulheres no mundo, representando 4,4\% mortes relacionadas ao câncer ${ }^{1}$. Em Regiốes desenvolvidas, o risco médio de morrer por câncer de ovário antes dos 75 anos é duas vezes maior do que nas Regióes menos desenvolvidas, com uma incidência superior a 7,5 por 100 mil habitantes ${ }^{2}$.

$\mathrm{Na}$ América Latina, o câncer é a segunda causa de morte, e as projeçóes mostram que essa causa poderá crescer consideravelmente entre 2012 e 2035 . Estima-se um crescimento de $91 \%$ de casos novos e de $106 \%$ no número de mortes, durante esse período, em consequência do envelhecimento e da mudança nos padróes de fecundidade nessa Região, embora sejam esperadas diferenças internas nos padrôes de incidência e mortalidade por câncer, o que pode estar relacionado diretamente com o padráo de desenvolvimento e aos distintos processos de transição demográfica e epidemiológica vivenciados por cada país dessa Regiáo $0^{3,4}$.

Entre os fatores associados ao câncer do ovário, destacam-se: história familiar, os reprodutivos (nuliparidade, lactação, uso de anticoncepcional oral, ligadura de trombas e ooforectomia) e aqueles relacionados aos hábitos e ao estilo de vida (tabagismo, aumento do consumo de carnes e gorduras, inatividade física) ${ }^{5-8}$.

Em razão do seu desenvolvimento silencioso, cerca de 3/4 dos cânceres de ovário apresentam-se em estágio avançado no momento do diagnóstico 9 . Um fator que dificulta o diagnóstico precoce é a falta de estratégias de rastreio de fácil acesso, visto que a forma mais prática para identificar a predisposição à doença são os testes genéticos que, além do alto custo, devem ser customizados com as variantes patogênicas mais frequentes em cada população, o que torna o acesso muito limitado ${ }^{10}$.

O controle do câncer tem se tornado um grande desafio na América Latina. O aumento da incidência, acompanhado por taxas de mortalidade desproporcionalmente altas quando comparadas às demais Regióes do mundo, revela a magnitude do desafio a ser enfrentado. Apesar de as neoplasias estarem entre as principais causas de morte nessa Regiáo, as estratégias de controle lidam com questóes como a organização e o desenvolvimento de serviços de saúde, mecanismos de formulação de políticas e programas e até mesmo a mobilização da sociedade ${ }^{11}$.

A descrição da distribuição geográfica de incidência e mortalidade de um câncer identifica o perfil da população e, consequentemente, seu padrão de qualidade de vida. Tendo em vista a dificuldade que os países da América Latina enfrentam no controle do câncer e em consolidar o diagnóstico precoce para o câncer de ovário, faz-se necessário conhecer a epidemiologia desse câncer na América Latina, com vistas a auxiliar na elaboração de novas políticas de enfrentamento direcionadas às áreas e aos grupos populacionais de maior vulnerabilidade.

Dessa forma, o objetivo deste estudo foi analisar as tendências da incidência e da mortalidade para o câncer de ovário em países da América Latina.

\section{MÉTODO}

Trata-se de um estudo ecológico de série temporal, baseado em dados secundários disponíveis nas bases de dados da Agência Internacional para Pesquisa do Câncer $(\operatorname{Iarc})^{2}$ e da Organização Mundial da Saúde (OMS) ${ }^{12}$. Foram analisadas as tendências da incidência e da mortalidade da neoplasia maligna de ovário, ocorridas nos países da América Latina.

Casos incidentes de neoplasia maligna de ovários, durante o período de 22 anos (1990-2012), foram extraídos da Cancer Incidence in Five Continents (CI5 $\mathrm{XI})$, que incluiu cinco Registros de Câncer de Base Populacional (RCBP): quatro registros regionais, Cali (Colômbia), Goiânia (Brasil), Quito (Equador), Valdivia (Chile); e um registro nacional, Costa Rica ${ }^{2}$. Para os dados de mortalidade, foram analisadas as informaçóes disponíveis de 17 países da América Latina (Argentina, Belize, Brasil, Chile, Colômbia, Costa Rica, Equador, El Salvador, Guatemala, México, Nicarágua, Panamá, Paraguai, Peru, Suriname, Uruguai e Venezuela), que representaram cerca de $90 \%$ da população entre 1995 a $2013^{13}$.

O número de casos foi extraído e as taxas específicas ajustadas por idade foram calculadas para três grupos etários (0-39; 40-59 e 60-74) e para todas as idades. A divisão por faixas etárias proposta neste estudo teve como objetivo categorizar os dados para uma melhor visualizaçáo dos grupos de maior carga da doença, com base na leitura prévia a respeito das faixas etárias de maior incidência e mortalidade. $\mathrm{E}$ todas as idades representam o cálculo da incidência e da mortalidade para o conjunto da população, sem divisão por faixas. As taxas específicas ajustadas por idade foram calculadas usando a população-padrão mundial de acordo com os países com dados disponíveis.

A variação percentual média anual (AAPC) foi estimada para incidência e mortalidade com um intervalo de confiança de 95\% (IC 95\%) no período. A exceção, para essas análises, foi Belize, Guatemala, Suriname e Uruguai, em virtude da falta de casos na série histórica. As análises estatísticas foram realizadas utilizando-se o software Joinpoint Regression Program, versão 4.5.0.0, conforme Kim et al ${ }^{14}$. 


\section{RESULTADOS}

Entre 1990 e 2012, as maiores taxas de incidência para o câncer de ovário foram observadas em Cali (Colômbia) e Goiânia (Brasil), na faixa etária de 60 a 74 anos, com taxas de 35,4 e 26,3 casos por 100 mil habitantes, respectivamente. Houve tendência de estabilidade na maioria dos países, exceto em Goiânia (Brasil), na faixa etária de 60 a 74 anos, que apresentou aumento (AAPC: 3,4\%; IC 95\%: 0,3; 6,6). As menores taxas de incidência foram observadas na faixa etária de 0 a 39 anos, com tendência de estabilidade para a coorte histórica (Tabela 1, Figura 1).

Entre 1995 e 2013, a maior taxa de mortalidade por câncer de ovário foi registrada no Uruguai (21,6 óbitos/100 mil hab.) para a faixa etária de 60 a 74 anos; e a menor em Belize e Guatemala (0,2 óbitos/100 mil hab.), para a faixa etária de 0 a 39 anos (Tabela 2).

Nos 13 países estudados (Tabela 3, Figura 2), para as tendências de mortalidade na faixa etária de 60 a 74 anos, apenas a Costa Rica apresentou redução (AAPC:
$-3,04 \%$; IC 95\%: -5,27; -0.76), os demais apresentaram aumento e estabilidade em suas tendências. Para a faixa etária de 40 a 59 anos, as tendências com aumento significativo ocorreram em El Salvador (AAPC: 5,80\%; IC 95\%: 3,01;8.67) e Panamá (AAPC: 5,18\%; IC 95\%: $1,87 ; 8.61)$. Nenhum país apresentou redução para essa faixa etária.

Para as tendências de mortalidade na faixa etária de 0 a 39 anos, houve aumento significativo na Argentina (AAPC: $0,28 \%$; IC 95\%: 1,$00 ; 1,58$ ) e no Brasil (AAPC: 0,80\%; IC 95\%: 0,26; 1,35). Os demais países apresentaram estabilidade.

\section{DISCUSSÃO}

A incidência por câncer de ovário na América Latina manteve-se estável ao longo da série analisada na faixa etária acima de 60 anos para a maioria dos países, com exceção do Brasil que registrou aumento. Entre as faixas etárias estudadas, a menor taxa de incidência foi apontada nas mulheres mais jovens, de 0 a 39 anos, e as maiores taxas

Tabela 1. Dados dos Registros de Câncer de Base Populacional em Cali (Colômbia), Costa Rica, Goiânia (Brasil), Quito (Equador) e Valdivia (Chile) período 1990-2012

\begin{tabular}{|c|c|c|c|c|c|}
\hline RCBP & $\begin{array}{c}\text { Dados } \\
\text { disponíveis }\end{array}$ & $\begin{array}{c}\text { Grupo por } \\
\text { idade }\end{array}$ & $\begin{array}{c}\text { Taxa de } \\
\text { incidência } \\
\text { padronizada }\end{array}$ & AAPC (IC 95\%) & p-valor \\
\hline \multirow{4}{*}{$\begin{array}{c}\text { Cali } \\
\text { (Colômbia) }\end{array}$} & $1990-2012$ & $0-39$ & 1,7 & $-1,2(-3,2 ; 0,8)$ & $p>0,05$ \\
\hline & & $40-59$ & 16,8 & $-1,0(-2,1 ; 0,1)$ & $p>0,05$ \\
\hline & & $60-74$ & 35,4 & $-1,3(-2,6 ; 0,1)$ & $p>0,05$ \\
\hline & & Total & 8,0 & $-1,1 \quad(-1,9 ;-0,4)$ & $p>0,05$ \\
\hline \multirow[t]{4}{*}{ Costa Rica } & $1990-2011$ & $0-39$ & 1,5 & $-0,1(-1,5 ; 1,3)$ & $p>0,05$ \\
\hline & & $40-59$ & 10,4 & $-0,7(-1,8 ; 0,5)$ & $p>0,05$ \\
\hline & & $60-74$ & 20,8 & $-0,6(-2,2 ; 1,0)$ & $p>0,05$ \\
\hline & & Total & 5,2 & $-0,6(-1,4 ; 0,3)$ & $p>0,05$ \\
\hline Goiânia & $1993-2012$ & $0-39$ & 1,4 & $5,9(-1,6 ; 13,9)$ & $p>0,05$ \\
\hline \multirow{3}{*}{ (Brasil) } & & $40-59$ & 13,5 & $0,3(-2,2 ; 2,8)$ & $p>0,05$ \\
\hline & & $60-74$ & 26,3 & $3,4(0,3 ; 6,6)$ & $p<0,05$ \\
\hline & & Total & 6,3 & $0,9(-3,1 ; 5,1)$ & $p>0,05$ \\
\hline \multirow{4}{*}{$\begin{array}{c}\text { Quito } \\
\text { (Equador) }\end{array}$} & $1990-2012$ & $0-39$ & 1,6 & $0,4(-2,6 ; 3,6)$ & $p>0,05$ \\
\hline & & $40-59$ & 15,8 & $0,5(-1,8 ; 2,8)$ & $p>0,05$ \\
\hline & & $60-74$ & 25,3 & $0,6(-1,3 ; 2,5)$ & $p>0,05$ \\
\hline & & Total & 6,8 & $0,3(-1,0 ; 1,7)$ & $p>0,05$ \\
\hline \multirow[t]{4}{*}{ Valdivia (Chile) } & $1998-2012$ & $0-39$ & 1,5 & $-4,4(-11,7 ; 3,4)$ & $p>0,05$ \\
\hline & & $40-59$ & 15,3 & $-1,6(-6,4 ; 3,5)$ & $p>0,05$ \\
\hline & & $60-74$ & 23,9 & $-3,3(-8,9 ; 2,7)$ & $p>0,05$ \\
\hline & & Total & 6,5 & $-2,8(-6,2 ; 0,7)$ & $p>0,05$ \\
\hline
\end{tabular}

Legendas: AAPC: Variação percentual média anual; IC: Intervalo de confiança; RCBP: Registros de Câncer de Base Populacional. Nota: Populaçăo-padrão mundial (ASW/100 mil hab.). 


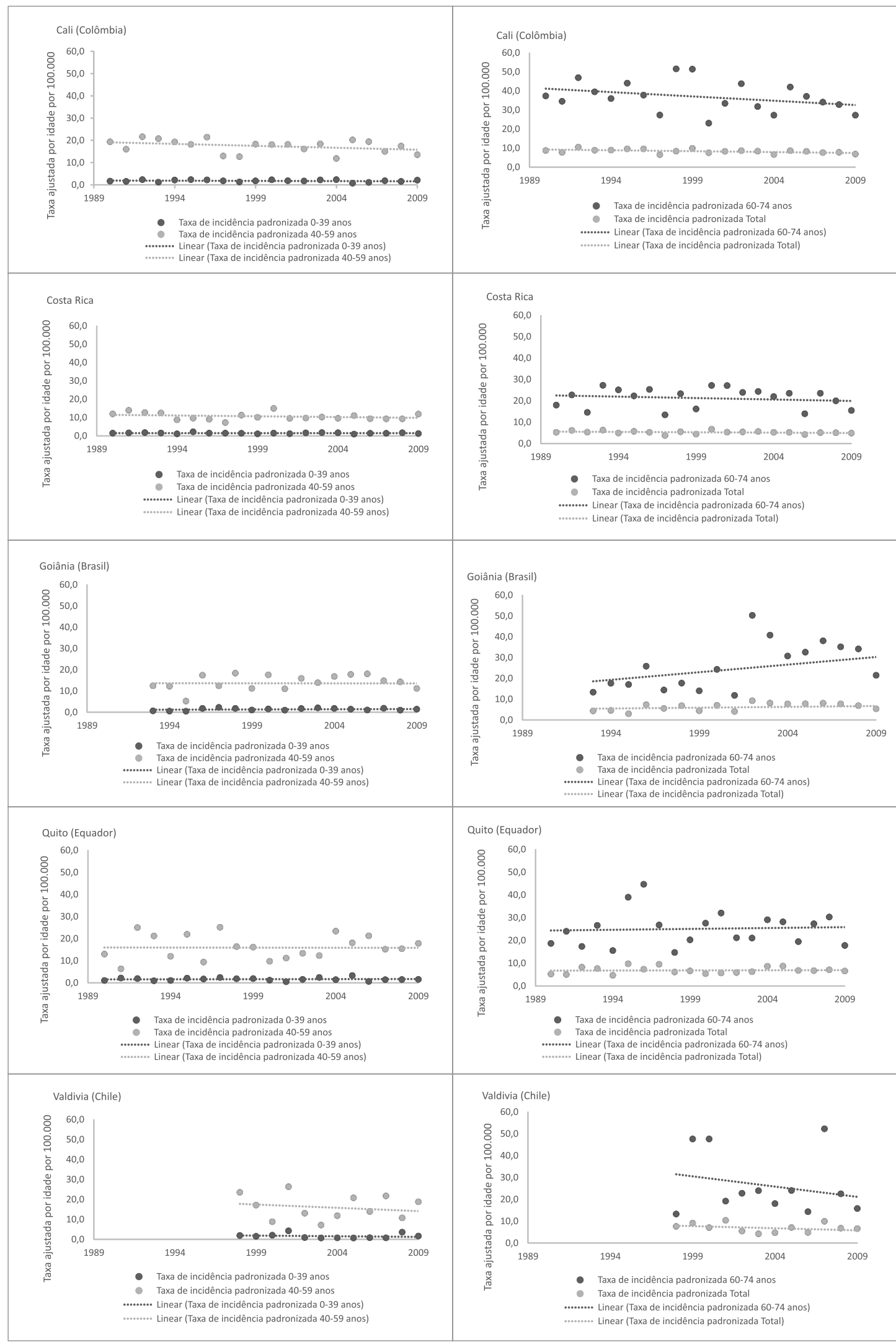

Figura 1. Taxa de incidência ajustada por idade 0-39 e 40-59 anos e acima de 60 anos e total, para Cali (Colômbia), Costa Rica, Goiânia (Brasil), Quito (Equador) e Valdivia (Chile), para câncer de ovário, período 1990-2012

Fonte: Dados extraídos do CI5plus ${ }^{2}$.

Legenda: IC 95\%: intervalo de confiança de $95 \%$.

Nota: A linha cinza representa as tendências do período. 
Tabela 2. Taxa de mortalidade padronizada por idade por 100 mil mulheres, número de óbitos para o câncer de ovário, por faixa etária, para 17 populações latino-americanas, período 1995-2013

\begin{tabular}{|c|c|c|c|}
\hline RCBP & Dados disponíveis & Grupos por idade & $\begin{array}{c}\text { Taxa de mortalidade } \\
\text { padronizada }\end{array}$ \\
\hline \multirow[t]{4}{*}{ Argentina } & $1997-2013$ & $0-39$ & 0,3 \\
\hline & & $40-59$ & 7,1 \\
\hline & & $60-74$ & 19,3 \\
\hline & & Total & 3,5 \\
\hline \multirow[t]{4}{*}{ Belize } & $1997-2013$ & $0-39$ & 0,2 \\
\hline & & $40-59$ & 2,9 \\
\hline & & $60-74$ & 3,1 \\
\hline & & Total & 1,1 \\
\hline \multirow[t]{4}{*}{ Brasil } & $1996-2013$ & $0-39$ & 0,3 \\
\hline & & $40-59$ & 4,4 \\
\hline & & $60-74$ & 12,6 \\
\hline & & Total & 2,3 \\
\hline \multirow[t]{4}{*}{ Chile } & $1997-2013$ & $0-39$ & 0,3 \\
\hline & & $40-59$ & 6,5 \\
\hline & & $60-74$ & 16,1 \\
\hline & & Total & 3,0 \\
\hline \multirow[t]{4}{*}{ Colômbia } & $1997-2013$ & $0-39$ & 0,3 \\
\hline & & $40-59$ & 5,4 \\
\hline & & $60-74$ & 14,8 \\
\hline & & Total & 2,7 \\
\hline \multirow[t]{4}{*}{ Costa Rica } & $1997-2013$ & $0-39$ & 0,3 \\
\hline & & $40-59$ & 4,2 \\
\hline & & $60-74$ & 13,1 \\
\hline & & Total & 2,3 \\
\hline \multirow[t]{4}{*}{ Equador } & $1997-2013$ & $0-39$ & 0,3 \\
\hline & & $40-59$ & 3,7 \\
\hline & & $60-74$ & 8,6 \\
\hline & & Total & 1,8 \\
\hline \multirow[t]{4}{*}{ El Salvador } & $1997-2013$ & $0-39$ & 0,3 \\
\hline & & $40-59$ & 3,1 \\
\hline & & $60-74$ & 6,7 \\
\hline & & Total & 1,5 \\
\hline \multirow[t]{4}{*}{ Guatemala } & $2000-2013$ & $0-39$ & 0,2 \\
\hline & & $40-59$ & 1,8 \\
\hline & & $60-74$ & 4,0 \\
\hline & & Total & 0,9 \\
\hline \multirow[t]{4}{*}{ México } & $1998-2013$ & $0-39$ & 0,4 \\
\hline & & $40-59$ & 5,3 \\
\hline & & $60-74$ & 14,8 \\
\hline & & Total & 3,0 \\
\hline \multirow[t]{4}{*}{ Nicarágua } & $1997-2013$ & $0-39$ & 0,3 \\
\hline & & $40-59$ & 3,1 \\
\hline & & $60-74$ & 7,6 \\
\hline & & Total & 1,6 \\
\hline \multirow[t]{4}{*}{ Panamá } & $1998-2013$ & $0-39$ & 0,3 \\
\hline & & $40-59$ & 3,8 \\
\hline & & $60-74$ & 12,4 \\
\hline & & Total & 2,2 \\
\hline
\end{tabular}


Tabela 2. continuação

\begin{tabular}{|c|c|c|c|}
\hline RCBP & Dados disponíveis & Grupos por idade & $\begin{array}{c}\text { Taxa de mortalidade } \\
\text { padronizada }\end{array}$ \\
\hline \multirow[t]{4}{*}{ Paraguai } & 1996-2013 & $0-39$ & 0,3 \\
\hline & & $40-59$ & 4,1 \\
\hline & & $60-74$ & 9,2 \\
\hline & & Total & 1,9 \\
\hline \multirow[t]{4}{*}{ Peru } & 1999-2013 & $0-39$ & 0,3 \\
\hline & & $40-59$ & 4,1 \\
\hline & & $60-74$ & 9,3 \\
\hline & & Total & 1,9 \\
\hline \multirow[t]{4}{*}{ Suriname } & $1995-2013$ & $0-39$ & 0,4 \\
\hline & & $40-59$ & 4,6 \\
\hline & & $60-74$ & 14,0 \\
\hline & & Total & 2,5 \\
\hline \multirow[t]{4}{*}{ Uruguai } & 1997-2013 & $0-39$ & 0,3 \\
\hline & & $40-59$ & 7,6 \\
\hline & & $60-74$ & 21,6 \\
\hline & & Total & 3,9 \\
\hline \multirow[t]{4}{*}{ Venezuela } & 1996-2013 & $0-39$ & 0,4 \\
\hline & & $40-59$ & 5,9 \\
\hline & & $60-74$ & 14,8 \\
\hline & & Total & 2,9 \\
\hline
\end{tabular}

Legenda: RCBP: Registros de Câncer de Base Populacional.

Nota: Não houve dados registrados em 2011 no Uruguai. População-padrão mundial (ASW/100 mil hab.).

Tabela 3. Tendências da mortalidade por câncer de ovário, por faixa etária, para 17 populações latino-americanas, período 1995 -2013

\begin{tabular}{|c|c|c|c|c|}
\hline RCBP & Dados disponíveis & Grupos por idade & AAPC (IC 95\%) & p-valor \\
\hline \multirow[t]{4}{*}{ Argentina } & 1997-2013 & $0-39$ & $0,28(1,00 ; 1,58)$ & $p<0,05$ \\
\hline & & $40-59$ & $0,03(0,53 ; 0,60)$ & $p<0,05$ \\
\hline & & $60-74$ & $-0,30(0,87 ; 0,27)$ & $\mathrm{p}<0,05$ \\
\hline & & Total & $-0,13(-0,51 ; 0,25)$ & $p>0,05$ \\
\hline \multirow[t]{4}{*}{ Belize } & 1997-2013 & $0-39$ & - & - \\
\hline & & $40-59$ & - & - \\
\hline & & $60-74$ & - & - \\
\hline & & Total & - & - \\
\hline \multirow[t]{4}{*}{ Brasil } & $1996-2013$ & $0-39$ & $0,80(0,26 ; 1,35)$ & $p<0,05$ \\
\hline & & $40-59$ & $0,34(0,03 ; 0,66)$ & $p<0,05$ \\
\hline & & $60-74$ & $0,56(0,18 ; 0,94)$ & $p<0,05$ \\
\hline & & Total & $0,48(0,25 ; 0,71)$ & $p<0,05$ \\
\hline \multirow[t]{6}{*}{ Chile } & $1997-2013$ & $0-39$ & $-2,10(-4,18 ; 0,03)$ & $p>0,05$ \\
\hline & & $40-59$ & $-1,56(-2,76 ; 0,34)$ & $p>0,05$ \\
\hline & & $60-74$ & $0,21(-0,55 ; 0,98)$ & $p>0,05$ \\
\hline & & Total & $-0,74(-1,50 ; 0,03)$ & $p>0,05$ \\
\hline & & $60-74$ & $-0,13(-0,77 ; 0,51)$ & $p>0,05$ \\
\hline & & Total & $0,40(-0,11 ; 0,90)$ & $p>0,05$ \\
\hline \multirow[t]{4}{*}{ Costa Rica } & $1997-2013$ & $0-39$ & $-0,59(-5,86 ; 4,97)$ & $p>0,05$ \\
\hline & & $40-59$ & $0,59(-1,67 ; 2,90)$ & $p>0,05$ \\
\hline & & $60-74$ & $-3,04(-5,27 ;-0,76)$ & $p>0,05$ \\
\hline & & Total & $-1,31(-2,47 ; 0,13)$ & $p>0,05$ \\
\hline
\end{tabular}


Tabela 3. continuação

\begin{tabular}{|c|c|c|c|c|}
\hline RCBP & Dados disponíveis & Grupos por idade & AAPC (IC 95\%) & p-valor \\
\hline \multirow[t]{4}{*}{ Equador } & 1997-2013 & $0-39$ & $-1,05(-2,79 ; 0,73)$ & $p>0,05$ \\
\hline & & $40-59$ & $0,03(-1,34 ; 1,43)$ & $p>0,05$ \\
\hline & & $60-74$ & $3,14(1,54 ; 4,76)$ & $p<0,05$ \\
\hline & & Total & $1,22(0,27 ; 2,19)$ & $p<0,05$ \\
\hline \multirow[t]{4}{*}{ El Salvador } & 1997-2013 & $0-39$ & $2,93(-2,13 ; 8,25)$ & $p>0,05$ \\
\hline & & $40-59$ & $5,80(3,01 ; 8,67)$ & $p<0,05$ \\
\hline & & $60-74$ & $4,11(0,71 ; 7,63)$ & $\mathrm{p}<0,05$ \\
\hline & & Total & $4,56(2,02 ; 7,15)$ & $p<0,05$ \\
\hline \multirow[t]{4}{*}{ Guatemala } & $2000-2013$ & $0-39$ & - & - \\
\hline & & $40-59$ & - & - \\
\hline & & $60-74$ & - & - \\
\hline & & Total & - & - \\
\hline \multirow[t]{4}{*}{ México } & $1998-2013$ & $0-39$ & $0,47(-0,63 ; 1,59)$ & $p>0,05$ \\
\hline & & $40-59$ & $0,89(0,53 ; 1,25)$ & $p<0,05$ \\
\hline & & $60-74$ & $1,41(0,90 ; 1,93)$ & $p<0,05$ \\
\hline & & Total & $1,09(0,75 ; 1,42)$ & $p<0,05$ \\
\hline \multirow[t]{4}{*}{ Nicarágua } & 1997-2013 & $0-39$ & $-1,96(-6,04 ; 2,29)$ & $p>0,05$ \\
\hline & & $40-59$ & $3,24(0,53 ; 6,02)$ & $p<0,05$ \\
\hline & & $60-74$ & $0,57(-2,20 ; 3,42)$ & $p>0,05$ \\
\hline & & Total & $1,17(-0,56 ; 2,93)$ & $p>0,05$ \\
\hline \multirow[t]{4}{*}{ Panamá } & 1998-2013 & $0-39$ & $0,82(-4,61 ; 6,55)$ & $p>0,05$ \\
\hline & & $40-59$ & $5,18(1,87 ; 8,61)$ & $p<0,05$ \\
\hline & & $60-74$ & $1,09(-1,21 ; 3,45)$ & $p>0,05$ \\
\hline & & Total & $2,41(0,17 ; 4,70)$ & $p<0,05$ \\
\hline \multirow[t]{4}{*}{ Paraguai } & 1996-2013 & $0-39$ & $0,45(-5,09 ; 6,30)$ & $p>0,05$ \\
\hline & & $40-59$ & $0,99(-0,94 ; 2,96)$ & $p>0,05$ \\
\hline & & $60-74$ & $3,19(-0,32 ; 6,82)$ & $p>0,05$ \\
\hline & & Total & $1,73(-0,13 ; 3,63)$ & $p>0,05$ \\
\hline \multirow[t]{4}{*}{ Peru } & 1999-2013 & $0-39$ & $1,81(-0,97 ; 4,66)$ & $p>0,05$ \\
\hline & & $40-59$ & $1,97(0,58 ; 3,37)$ & $p<0,05$ \\
\hline & & $60-74$ & $2,88(0,84 ; 4,96)$ & $\mathrm{p}<0,05$ \\
\hline & & Total & $2,30(0,97 ; 3,65)$ & $\mathrm{p}<0,05$ \\
\hline \multirow[t]{4}{*}{ Suriname } & 1995-2013 & $0-39$ & - & - \\
\hline & & $40-59$ & - & - \\
\hline & & $60-74$ & - & \\
\hline & & Total & $7,19(0,82 ; 13,95)$ & $\mathrm{p}<0,05$ \\
\hline \multirow[t]{2}{*}{-} & & $60-74$ & - & - \\
\hline & & Total & & - \\
\hline \multirow[t]{4}{*}{ Venezuela } & 1996-2013 & $0-39$ & $-0,77(-2,07 ; 0,54)$ & $p>0,05$ \\
\hline & & $40-59$ & $0,72(-0,20 ; 1,65)$ & $p>0,05$ \\
\hline & & $60-74$ & $0,54(-0,11 ; 1,20)$ & $p>0,05$ \\
\hline & & Total & $0,46(0,01 ; 0,92)$ & $p<0,05$ \\
\hline
\end{tabular}

Legendas: AAPC: Variação percentual média anual; IC: Intervalo de confiança; RCBP: Registros de Câncer de Base Populacional.

Nota: População-padrão mundial (ASW/100 mil hab.).

para as maiores de 60 anos, que podem ser justificadas pelas mudanças ocorridas nos padróes reprodutivos (aumento da idade no primeiro parto e menor paridade) e aumento da expectativa de vida ${ }^{4}$.

Estudos corroboram que o câncer de ovário possui elevadas taxas de incidência em outros lugares do mundo, não sendo um destaque apenas da América Latina. $\mathrm{Na}$ América Central, esse câncer ocupou a oitava posição entre os cânceres mais frequentes em mulheres no ano 2012, com uma incidência de cinco casos a cada 100 mil mulheres nas Regiôes menos desenvolvidas e 9,1 casos/100 mil mulheres nas Regiōes mais desenvolvidas. 


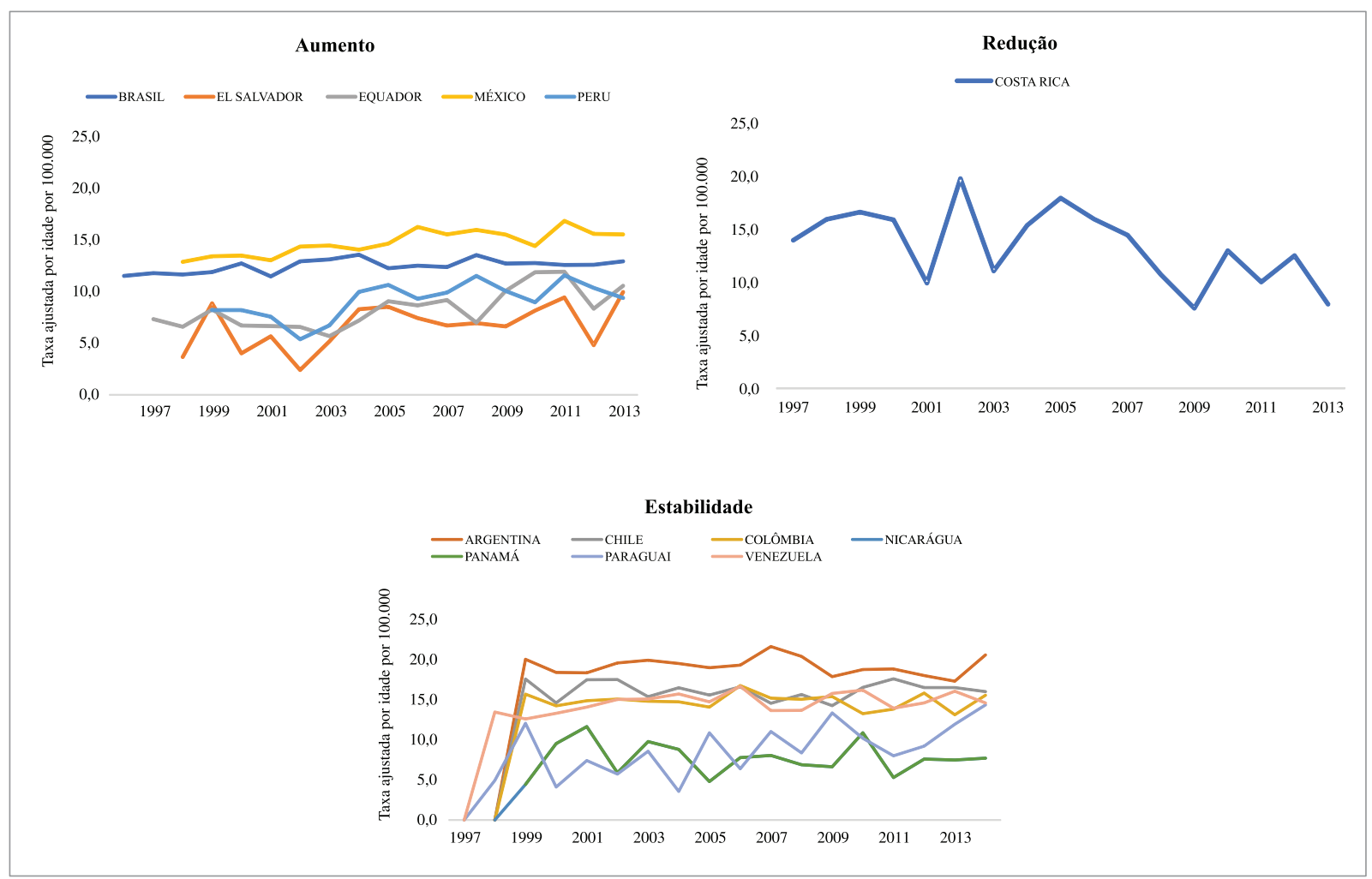

Figura 2. Tendência temporal da mortalidade por câncer de ovário, para o grupo de mulheres com idade acima de 60 anos, em 13 países da América Latina, período 1995-2013

Fonte: Dados extraídos do WHO Mortality Database ${ }^{12}$.

$\mathrm{Na}$ América do Sul, ocupou a sétima posição, com uma incidência de 5,8 casos/100 mil mulheres nas Regióes menos desenvolvidas e 9,1 casos/100 mil mulheres nas mais desenvolvidas ${ }^{15,4}$.

Ferlay et al. ${ }^{13}$ apontam uma estimativa da incidência das neoplasias ovarianas de 12,9 casos a cada 100 mil mulheres nos países europeus em 2018. As maiores incidências foram observadas na Sérvia $(21,8$ casos/100 mil mulheres) e Belarus (20,1 casos/100 mil mulheres); e a menor incidência foi registrada para a Albânia $(5,1$ casos para cada 100 mil mulheres). Estudos ressaltam que países com Índice de Desenvolvimento Humano (IDH) alto/muito alto possuem uma maior incidência para câncer de ovário quando comparados aos países de médio/baixo $\mathrm{IDH}^{1}$.

Segundo Souza et al. ${ }^{15}$, aproximadamente $70 \%$ dos tumores ovarianos ocorrem em idade reprodutiva, com maior incidência entre 21 e 40 anos. No presente estudo, evidenciou-se um maior número de casos incidentes na América Latina para a faixa etária de 40 a 59 anos. Utilizando dados de prevalência cumulativa, estudos mostram que a probabilidade de uma mulher ter câncer de ovário entre o nascimento até os 85 anos é de 1,5\%, e a sobrevida global é de $30 \%$ a $40 \%$ em cinco anos ${ }^{16-18}$.
Apesar de a incidência geral do câncer ser mais baixa na América Latina, comparada aos países europeus e aos Estados Unidos, a mortalidade por esse câncer é maior ${ }^{19}$. A análise de mortalidade entre os países estudados aponta a faixa etária acima de 60 anos como a que mais registra mortes por câncer de ovário, com destaque para o Uruguai, com a maior taxa de mortalidade para a faixa etária de 60 a 74 anos.

No Uruguai, o câncer de ovário é o sétimo mais prevalente e o sexto que mais provoca óbitos entre todos os cânceres em mulheres ${ }^{20}$. Embora estudos divulgados na Economist Intelligence Unit ${ }^{3}$ aponte o Uruguai como o país que possui o melhor programa para o controle do câncer na América Latina, este aparece como um dos países com as menores taxas de fecundidade da América Latina.

Vale ainda destacar as tendências de mortalidade na faixa etária de 0 a 39 anos, com tendência de aumento significativo no Brasil (AAPC: 0,8\%; IC 95\%: 0,26; 1,35) e na Argentina (AAPC: 0,28\%; IC 95\%: 1,00; 1,58).

Em um estudo realizado com dados do Sistema de Informaçōes sobre Mortalidade (SIM) do Brasil, os pesquisadores apontaram que, no período de 1980 a 2014, a taxa média de mortalidade por câncer de ovário foi de 4,91 óbitos por câncer do ovário por 100 mil mulheres, 
com aumento progressivo da mortalidade com o avançar da idade em todas as Regióes brasileiras. Além disso, este estudo evidenciou ainda um padrão heterogêneo na evolução temporal da mortalidade por câncer do ovário nas Regiōes geográficas brasileiras, com um risco positivo e significativo de morte para as coortes mais antigas no Sul e Sudeste, e risco reduzido para as coortes mais jovens, sendo o inverso para as Regiôes Norte e Nordeste ${ }^{21}$.

Nesse sentido, no Brasil, torna-se necessário repensar as açôes de prevenção do câncer de ovário, embora se notem esforços no combate ao câncer a partir da publicação da Portaria n. ${ }^{\circ} 2.439$, de 8 de dezembro de $2005^{22}$, revogada pela Portaria n. ${ }^{\circ} 874$, de 16 de maio de $2013^{23}$, que institui a Política Nacional para a Prevenção e Controle do Câncer (PNPCC) na Rede de Atenção à Saúde das Pessoas com Doenças Crônicas (RASPDC) no âmbito do Sistema Único de Saúde (SUS). Entre seus componentes, estão a promoção e a vigilância em saúde, com vistas, sobretudo, às açôes que proporcionem a redução de fatores de risco para as neoplasias. Essa medida ainda não foi suficiente para observar mudanças ao longo da década seguinte, conforme refletem os dados do presente estudo, o que alerta para a revisão dessa Portaria e possíveis déficits na sua execução ${ }^{22-24}$.

O panorama da incidência e mortalidade por câncer de ovário na América Latina permite refletir ainda acerca da atual situação dos sistemas de saúde, que geralmente possuem estruturas fragmentadas que fornecem cuidados mínimos e, às vezes, auxiliam apenas as emergências. Adicionalmente, a atual crise global reverbera no planejamento e no orçamento destinados à saúde. Além disso, a alocação desigual de recursos humanos, a concentração de profissionais de saúde nos grandes centros urbanos e a falta de investimento em equipamentos e infraestrutura levam à reprodução das desigualdades socioeconômicas no cuidado de indivíduos com câncer, a exemplo do câncer de ovário; contribuindo, portanto, para consolidação dos aspectos epidemiológicos apresentados no presente estudo ${ }^{11}$.

A partir disso, pode-se inferir que os dados de incidência e mortalidade por câncer na América latina podem ser reflexos dos problemas na organizaçáo dos programas de controle do câncer, da qualidade dos serviços ofertados, bem como na infraestrutura dos serviços de saúde e no quantitativo de profissionais especializados reduzidos, o que pode influenciar na continuidade e integração do cuidado e na contrarreferência com outros serviços de saúde, resultando na dificuldade de diagnóstico precoce e consequentemente diminuindo as chances de cura em alguns cânceres, especialmente o câncer de ovário ${ }^{4}$.

A limitação identificada neste estudo é a possibilidade de haver subnotificação. Embora o número de RCBP incluídos na CI5plus tenha aumentado nos últimos anos, alguns países ainda não atendem aos padrões de qualidade e inserção dos dados, como é o caso de Belize, Guatemala, Suriname e Uruguai, que não tiveram suas tendências de mortalidade calculadas por falta de casos registrados na série histórica. $\mathrm{O}$ sub-registro de pacientes com câncer pode levar à subestimação das taxas de incidência e mortalidade, bem como dificultar nas avaliaçôes dos programas de controle e combate ao câncer. Apesar dessas limitaçóes, os dados utilizados no presente estudo foram validados por organizaçôes internacionais e podem ser usados para descrever a mortalidade nos países latino-americanos.

\section{CONCLUSÃO}

As altas taxas do câncer de ovário em alguns países da América Latina podem refletir deficiências na detecção precoce e controle da doença. Este estudo analisou a incidência do câncer de ovário a partir dos RCBP de quatro cidades e de um país, bem como as tendências de 12 países da América Latina.

Ao analisar a incidência de câncer de ovário, evidenciaram-se tendências de estabilidade para alguns países, como o Equador. No entanto, no Brasil, observou-se um aumento para as mulheres acima de 60 anos. Já para a mortalidade, verificou-se uma inclinação para estabilidade na maioria dos países da América Latina, como Argentina; enquanto o Brasil apresentou uma tendência de aumento para essa variável.

$O$ presente estudo fornece uma visáo relevante sobre o perfil epidemiológico do câncer de ovário e contribui para a identificaçáo da relevância e magnitude desse agravo para a população da América Latina. Essas análises auxiliam na elaboração de políticas que possam ser implementadas e direcionadas às áreas de maior vulnerabilidade.

\section{CONTRIBUIÇÕES}

Maria Aparecida Paulo dos Santos, Fábia Cheyenne Gomes de Morais Fernandes e Isabelle Ribeiro Barbosa contribuíram substancialmente na concepção e no planejamento do estudo, bem como na obtenção, análise e/ou interpretaçáo dos dados. Emelynne Gabrielly de Oliveira Santos e Dyego Leandro Bezerra de Souza contribuíram na redação e/ou revisão crítica. Todos os autores aprovaram a versão final a ser publicada.

\section{DECLARAÇÃO DE CONFLITO DE INTERESSES}

Nada a declarar. 


\section{FONTES DE FINANCIAMENTO}

Coordenação de Aperfeiçoamento de Pessoal de Nível Superior - Brasil (Capes) - Código de financiamento 001. Conselho Nacional de Desenvolvimento Científico e Tecnológico (CNPq), Programa Institucional de Bolsas de Iniciação Científica (Pibic).

\section{REFERÊNCIAS}

1. Bray F, Ferlay J, Soerjomataram I, et al. Global cancer statistics 2018: GLOBOCAN estimates of incidence and mortality worldwide for 36 cancers in 185 countries. Ca Cancer J Clin. 2018;68(6):394-424. doi: https://doi. org/10.3322/caac. 21492

2. Ferlay J, Colombet M, Bray F, editors. Cancer incidence in five continents, CI5plus. Lyon: International Agency for Research on Cancer; 2018.

3. Kielstra P. Controle do câncer, acesso e desigualdade na América Latina: uma história de luz e sombra [Internet]. [Londres]: Economist Intelligence Unit; 2017 [acesso 2019 mar 7]. Disponível em: https:// www.iccp-portal.org/sites/default/files/resources/ Cancer_control_access_and_inequality_in_Latin_ America_PORTUGUESE.pdf

4. Sierra MS, Soerjomataram I, Antoni S, et al. Cancer patterns and trends in Central and South America. Cancer Epidemiol. 2016;44(Suppl 1):S23-S42. doi: https://doi.org/10.1016/j.canep.2016.07.013

5. Meira KC, Santos J, Silva CMFP, et al. Efeitos da idade-período e coorte na mortalidade por câncer do ovário no Brasil e suas grandes regióes. Cad Saúde Pública. 2019;35(3). doi: http://doi.org/10.1590/0102311X00087018

6. Instituto Nacional de Câncer José Alencar Gomes da Silva. Estimativa 2020: incidência de câncer no Brasil [Internet]. Rio de Janeiro: INCA; 2019 [acesso 2019 jun 13]. Disponível em: https://www.inca.gov. br/publicacoes/livros/estimativa-2020-incidencia-decancer-no-brasil

7. Instituto Nacional de Câncer José Alencar Gomes da Silva [Internet]. Rio de Janeiro: INCA; [data desconhecida]. Tipos de câncer: câncer de ovário; 2019 [acesso 2019 mar. 05]. Disponível em: https://www.inca.gov.br/tiposde-cancer/cancer-de-ovario

8. Böhm-Vélez M, Mendelson E, Bree R, et al. Critérios de adequação do ACR: rastreamento do câncer de ovário [Internet]. São Paulo: Colégio Brasileiro de Radiologia e Diagnóstico por Imagem; 2017 [acesso $2018 \mathrm{dez}$ 11]. Disponível em: https://cbr.org.br/wp-content/ uploads/2017/06/04_06v2..pdf

9. Oliveira KM, Oliveira MM, Araújo RS. Câncer de ovário e detecção precoce: revisão bibliográfica da literatura. Rev Cient Facmais. 2016;7(3):58-65.
10. Temes BAB. Síndromes de predisposição hereditária ao câncer de mama e/ou ovário: análises genômicas, epidemiologia molecular e caracterização clínica [tese]. Porto Alegre, RS: Universidade Federal do Rio Grande do Sul; 2017.

11. Curado MP, de Souza DLB. Cancer burden in Latin America and the Caribbean. Ann Glob Health. 2014;80(5):370-7. doi: https://doi.org/10.1016/j. aogh.2014.09.009

12. WHO Mortality Database [Internet]. Geneva: World Health Organization; [data desconhecida] - [updated 2018 May; cited 2019 Mar 26]. Available from: https:// www.who.int/healthinfo/mortality_data/en/

13. Ferlay J, Colombet M, Soerjomataram I, et al. Cancer incidence and mortality patterns in Europe: estimates for 40 countries and 25 major cancers in 2018. Eur J Cancer. 2018;103:356-87. doi: https://doi.org/10.1016/j. ejca.2018.07.005

14. Kim HJ, Fay MP, Feuer EJ, et al. Permutation tests for joinpoint regression with applications to cancer rates. Stat Med. 2000;19(3):335-51. doi: https://doi. org/10.1002/(sici) 1097-0258(20000215)19:3<335::aid$\operatorname{sim} 336>3.0 . \mathrm{co} ; 2-\mathrm{z}$

15. Souza E, Yoshida A, Peres H, et al. Preservação da fertilidade e dos ovários em mulheres com tumores anexiais benignos. Rev Bras Ginecol Obstetr. 2015;37(1):36-41. doi: http://doi.org/10.1590/SO100-720320140005179

16. Barbosa IR. Tendências e projeçóes da mortalidade pelos cânceres específicos ao gênero no Brasil [tese]. Natal, RN: Universidade Federal do Rio Grande do Norte; 2015.

17. Silva AP, Venâncio TT, Figueiredo-Alves RR. Câncer ginecológico e gravidez: uma revisão sistematizada direcionada para obstetras. Femina. 2015;43(3):111-18.

18. Associação Brasileira para o Estudo da Obesidade e da Síndrome Metabólica. Mapa da obesidade [Internet]. São Paulo: Abeso; 2018 [acesso 2019 maio 24]. Disponível em: https://abeso.org.br/obesidade-esindrome-metabolica/mapa-da-obesidade/

19. Goss PE, Lee BL, Badovinac-Crnjevic T, et al. Planejamento do controle do câncer na América Latina e no Caribe. Lancet Oncol. 2013;14(5):391-436. doi: https://doi.org/10.1016/S1470-2045(13)70048-2

20. Barrios E, Garau M. Cáncer: magnitud del problema en el mundo y en Uruguay, aspectos epidemiológicos. Anfamed. 2017;4(1):7-161. doi: http://doi.org/10.25184/ anfamed2017.4.1.2

21. Meira KC, Santos J, CMFP Silva, et al. Efeitos da idade-período e coorte na mortalidade por câncer do ovário no Brasil e suas grandes regióes. Cad Saúde Pública. 2019;35(3):e00087018. doi: http://doi. org/10.1590/0102-311X00087018

22. Ministério da Saúde (BR). Portaria no 2.439, de 8 de dezembro de 2005. Institui a Política Nacional de Atenção Oncológica: Promoção, Prevenção, Diagnóstico, 
Tratamento, Reabilitaçáo e Cuidados Paliativos, a ser implantada em todas as unidades federadas, respeitadas as competências das três esferas de gestão. Diário Oficial da União. 2005 dez 9; Seção 1:80.

23. Ministério da Saúde (BR). Portaria no 874, de 16 de maio de 2013. Institui a Política Nacional para a Prevenção e Controle do Câncer na Rede de Atenção à Saúde das Pessoas com Doenças Crônicas no âmbito do Sistema Único de Saúde (SUS). Diário Oficial da União. 2013 maio 17; Seção 1:129.

24. Timoteo ARS. Identificação e caracterização molecular de mutações germinativas em indivíduos com síndrome de câncer de mama e ovário hereditário [tese]. Natal, RN: Universidade Federal do Rio Grande do Norte; 2016.

Recebido em 15/12/2019

Aprovado em 4/6/2020 